\title{
Incorporação de cinza de lenha, lodo de estação de tratamento de água e cinza de casca de arroz em massa cerâmica. Utilização da técnica de planejamento
}

\section{(Grog incorporation in ceramic mass to manufacture of bricks. A study of the physical-mechanical properties)}

\author{
E. N. M de Medeiros, R. M. Sposto, G. de A. Neves, R. R. Menezes \\ Programa de Pós-Graduação em Estruturas e Construção Civil, Departamento de Engenharia Civil e \\ Ambiental, Faculdade de Tecnologia, Universidade de Brasília - UnB, Campus Universitário Darcy Ribeiro \\ Brasília, DF \\ elisandra@unb.br
}

\begin{abstract}
Resumo
Este trabalho apresenta um estudo das propriedades físico-mecânicas de massas cerâmicas com a incorporação de resíduos de cinza de lenha, lodo de estação de tratamento de água e cinza de casca de arroz, visando a sua futura utilização na produção de blocos. Os procedimentos metodológicos consistiram na caracterização química e mineralógica, na análise granulométrica e no delineamento de misturas com argila e resíduos por meio da técnica de planejamento. Foram produzidos corpos de prova por prensagem, com variações de composições no intervalo de 0 a $50 \%$ de resíduo e 50 a $100 \%$ de argila. A queima foi realizada em forno tipo mufla. As propriedades físico-mecânicas avaliadas foram absorção de água e módulo de ruptura à flexão. Como resultados principais, concluiu-se sobre a viabilidade em se utilizar composições com até $25 \%$ de resíduo nas temperaturas de 800,900 e $1000{ }^{\circ} \mathrm{C}$.

Palavras-chave: cinza de lenha, cinza de casca de arroz, lodo de estação de tratamento de água, cerâmica vermelha.
\end{abstract}

\begin{abstract}
This paper presents a study of the physical and mechanical properties of ceramic mass with the incorporation of waste ash wood, sludge from water treatment and rice husk ash to determine whether its future use manufacture of bricks. The procedures consisted in the chemical and mineralogical characterization, particle size analysis and design of mixtures with clay and waste through planning technique. Bodies were produced by uniaxial pressing, with variations of compositions in the range of 0 to $50 \%$ waste and 50 to 100\% clay. The burning was carried out in muffle furnace. The physical and mechanical properties evaluated were water absorption and modulus of rupture strength. The main result, it was concluded on the feasibility of using compositions with up to $25 \%$ of waste at temperatures of 800,900 and $1000^{\circ} \mathrm{C}$.
\end{abstract}

Keywords: wood ash, rice husk ash, sludge from water treatment, bricks.

\section{INTRODUÇÃO}

As pesquisas sobre reciclagem e incorporação de resíduos industriais em novos produtos ou componentes têm se intensificado nos últimos anos, no Brasil e no Exterior. A principal justificativa na utilização destes resíduos é a minimização dos problemas ambientais e econômicos causados pela sua disposição, tais como: comprometimento estético da paisagem, ocupação de espaço, custo do recolhimento-processamento e degradação dos recursos naturais. Além disto, há que se considerar a possibilidade da diversificação de novos produtos no mercado com diminuição do custo final.

Para a viabilidade de utilização de resíduos na construção civil deve-se considerar, entre outros critérios, a sua localização e abundância [1]. Além disto, a inovação (a partir da incorporação de resíduos em novos produtos ou componentes) pode se transformar em oportunidade, se comprovado o desempenho dos novos produtos. O uso de resíduos em massas cerâmicas, tais como chamote, vidro, pó de rocha e outros, visando à obtenção de artefatos, tijolos e blocos, freqüentemente tem se mostrado uma boa solução para a minimização dos problemas ambientais.

Neste trabalho foram investigados três tipos de resíduos como adição em massa cerâmica para a produção de blocos. Os resíduos estudados são: a cinza da casca de arroz (CCA), o lodo de estação de tratamento de água (LETA) e a cinza da lenha (CL), cujas quantidades estimadas são apresentadas a seguir. Para a CCA, considerando-se uma produção de casca de arroz superior a 13 milhões de toneladas (o Brasil ocupa o nono lugar na lista dos produtores mundiais), é gerado um volume de cerca de $20 \%$ [2-5]. Em relação ao LETA, 
observa-se o seu aumento nos decantadores [6], e estima-se que são lançados em cursos d'água cerca de 2.000 toneladas/ dia de lodo sem nenhum tratamento [7]. E por fim, para a CL, estima-se uma geração mensal de aproximadamente 300 toneladas de cinzas em Campos dos Goytacazes, município situado no norte do Estado do Rio de Janeiro [8].

Este trabalho tem como objetivo realizar o delineamento de misturas que visam melhorar a atual situação das zonas industriais, utilizando CCA, LETAe CL. Foram determinadas as seguintes propriedades: absorção de água (AA) e módulo de ruptura à flexão (MRF), de acordo com a sistemática proposta em [9], utilizando-se técnicas de delineamento experimental e otimização, para avaliar o efeito simultâneo das características e conteúdo de argila e dos resíduos nas propriedades de composições para cerâmica vermelha.

\section{MATERIAIS E MÉTODOS}

A argila foi coletada de uma indústria de cerâmica vermelha da cidade de São Sebastião no Distrito Federal, com produção em torno de 500.000 blocos cerâmicos por mês. A jazida localiza-se a cerca de $10 \mathrm{~km}$ de distância da indústria. A cinza de casca de arroz foi coletada em uma empresa situada em Aparecida de Goiânia, GO, localizada a aproximadamente $220 \mathrm{~km}$ de Brasília, com produção de 25 toneladas por hora (capacidade atual de beneficiamento). O lodo foi coletado na ETA-Descoberto, sendo a principal estação de tratamento de água do sistema operado pela CAESB, no Distrito Federal, tratando uma vazão média de $3.800 \mathrm{~L} / \mathrm{s}$. A cinza de lenha foi coletada de uma indústria cerâmica da cidade de São Sebastião, DF, responsável pela fabricação de cerâmica branca da região.

A caracterização da amostra de argila estudada nesta pesquisa foi efetuada através dos seguintes métodos: análise granulométrica por difração de laser, análise química por espectrofotometria de fluorescência de raios X e difração de raios X com radiação Cuk $\alpha$ ( 40 kV, 30 mA). Para realização dos ensaios de caracterização, os resíduos foram passados em peneira ABNT $200(0,074 \mathrm{~mm})$, exceto para o ensaio de granulometria. Os resíduos foram beneficiados em moinho de bolas e passados em peneira ABNT 200, acondicionados em porta-amostra para análise por difração de raios X.

As composições foram formuladas utilizando-se planejamento experimental através da metodologia de delineamento de misturas. O desenvolvimento das composições A e B foi efetuado com um delineamento simplex simples aumentado com ponto central, utilizandose restrições no teor de matérias-primas para obtenção de blocos cerâmicos. Foram formuladas composições, segundo a metodologia de delineamento de misturas do planejamento experimental, contendo, na composição A: argila, LETA e CCA e na composição B: argila, CL e CCA, para avaliar a viabilidade da mistura dessas matérias-primas para produção de blocos cerâmicos. Foram estabelecidos limites inferiores e superiores de 50 e $100 \%$ de argila, respectivamente, e limites inferiores e superiores de 0 e $50 \%$ de resíduos, respectivamente.

Na Tabela I são apresentadas as composições A e B obtidas, tanto em termos dos componentes originais, como dos pseudocomponentes, das formulações para a produção de blocos cerâmicos. Os ensaios foram realizados em três replicatas. As composições foram obtidas através do planejamento em rede simplex $\{3,2\}$ com ponto central para as formulações. Foram utilizadas três replicatas para o desenvolvimento do trabalho.

As Figs. 1 e 2 mostram graficamente as composições formuladas na Tabela I.

As matérias-primas foram misturadas em moinho de bolas. Após a etapa de mistura, as massas foram secas e passadas em peneiras ABNT 80. Em seguida, foram confeccionados corpos-de-prova com molde de dimensões de $60 \mathrm{~mm}$ x $20 \mathrm{~mm}$ x $5 \mathrm{~mm}$ por prensagem uniaxial a $27 \mathrm{MPa}$. A secagem das peças foi realizada em estufa a $100{ }^{\circ} \mathrm{C}$ durante $24 \mathrm{~h}$. Após a secagem, os corpos de prova foram submetidos à queima, em ciclos de aquecimento de $8 \mathrm{~h}$ com patamar de queima de $2 \mathrm{~h}$ nas temperaturas de 800,900 e $1000{ }^{\circ} \mathrm{C}$, e

Tabela I - Composições A e B obtidas através do planejamento em rede simplex $\{3,2\}$.

[Table I-Compositions A and B of the design mixtures created by the augmented $\{3,2\}$ simplex lattice.]

\begin{tabular}{ccccccc}
\hline & \multicolumn{7}{c}{ Proporção (\%) } \\
\cline { 2 - 7 } Formulações & \multicolumn{7}{c}{ Composição A } & \multicolumn{3}{c}{ Composição B } \\
\cline { 2 - 7 } & Argila & LETA & CCA & Argila & CL & CCA \\
\hline \multirow{2}{*}{1} & 100,00 & 0,00 & 0,00 & 100,00 & 0,00 & 0,00 \\
2 & 50,00 & 50,00 & 0,00 & 50,00 & 50,00 & 0,00 \\
3 & 50,00 & 0,00 & 50,00 & 50,00 & 0,00 & 50,00 \\
4 & 75,00 & 25,00 & 0,00 & 75,00 & 25,00 & 0,00 \\
5 & 75,00 & 0,00 & 25,00 & 75,00 & 0,00 & 25,00 \\
6 & 50,00 & 25,00 & 25,00 & 50,00 & 25,00 & 25,00 \\
7 & 66,67 & 16,67 & 16,67 & 66,67 & 16,67 & 16,67 \\
8 & 83,33 & 8,33 & 8,33 & 83,33 & 8,33 & 8,33 \\
9 & 58,33 & 33,33 & 8,33 & 58,33 & 33,33 & 8,33 \\
10 & 58,33 & 8,33 & 33,33 & 58,33 & 8,33 & 33,33 \\
\hline
\end{tabular}




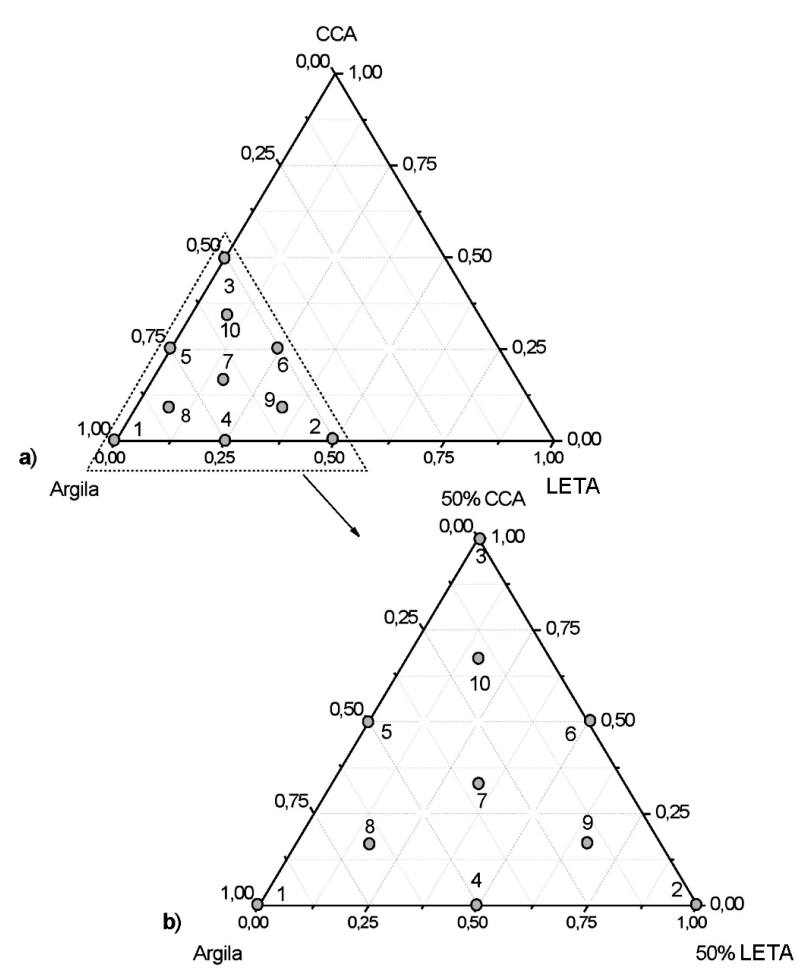

Figura 1: Esquema das formulações no diagrama ternário para a composição A.

[Figure 1: Schematic formulations in ternary diagram to composition A.]

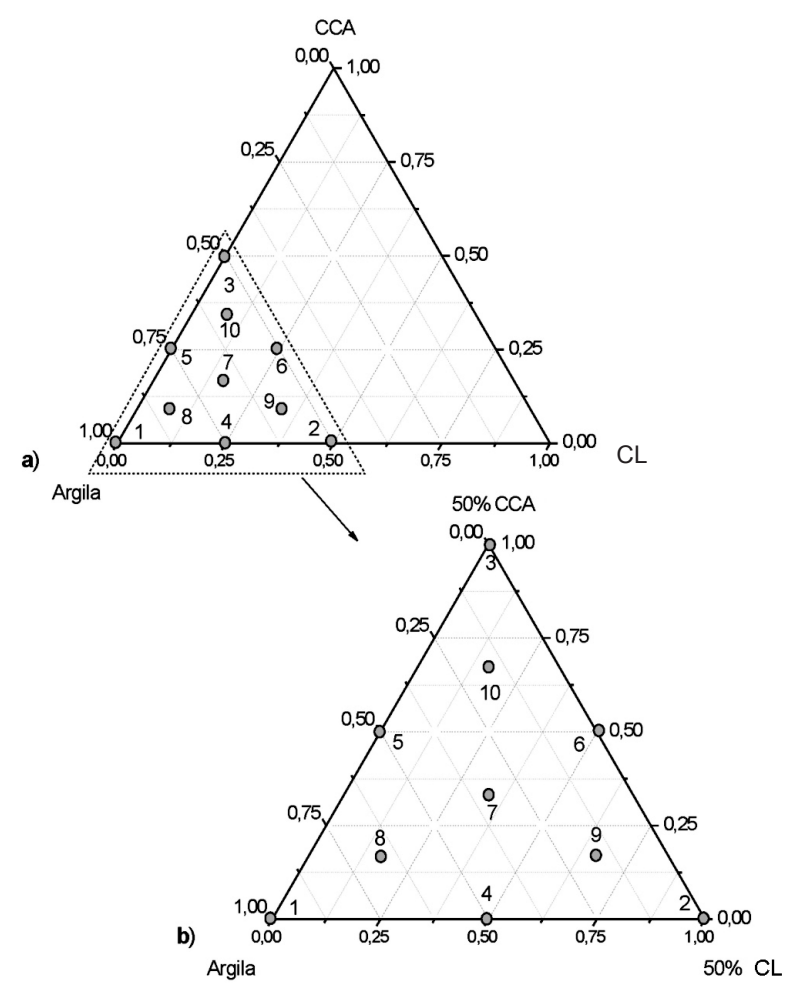

Figura 2: Esquema das formulações no diagrama ternário para a composição B.

[Figure 2: Schematic formulations in ternary diagram to composition B.] taxa de aquecimento de $2{ }^{\circ} \mathrm{C} / \mathrm{min}$ para que se obtivessem as condições de queima da indústria. Em seguida, para ambas as composições A e B, foram utilizados durante os ajustes dos valores de resposta (da AA, do MRF e da RQ) os modelos linear (equação $\mathrm{A}$ ), quadrático $(\mathrm{B})$, cúbico especial (equação C) e cúbico (D) em termos dos componentes $\mathrm{A}, \mathrm{G}$ e C.

$\mathrm{Y}(\mathrm{A}, \mathrm{G}, \mathrm{C})=\mathrm{b}_{1} \mathrm{~A}+\mathrm{b}_{2} \mathrm{G}+\mathrm{b}_{3} \mathrm{C}$

$\mathrm{Y}(\mathrm{A}, \mathrm{G}, \mathrm{C})=\mathrm{b}_{1} \mathrm{~A}+\mathrm{b}_{2} \mathrm{G}+\mathrm{b}_{3} \mathrm{C}+$ $\mathrm{b}_{4} \mathrm{AG}+\mathrm{b}_{5} \mathrm{AC}+\mathrm{b}_{6} \mathrm{GC}$

$\mathrm{Y}(\mathrm{A}, \mathrm{G}, \mathrm{C})=\mathrm{b}_{1} \mathrm{~A}+\mathrm{b}_{2} \mathrm{G}+\mathrm{b}_{3} \mathrm{C}+\mathrm{b}_{4} \mathrm{AG}+$ $\mathrm{b}_{5} \mathrm{AC}+\mathrm{b}_{6} \mathrm{GC}+\mathrm{b}_{7} \mathrm{AGC}$

$\mathrm{Y}(\mathrm{A}, \mathrm{G}, \mathrm{C})=\mathrm{b}_{1} \mathrm{~A}+\mathrm{b}_{2} \mathrm{G}+\mathrm{b}_{3} \mathrm{C}+\mathrm{b}_{4} \mathrm{AG}+$ $\mathrm{b}_{5} \mathrm{AC}+\mathrm{b}_{6} \mathrm{GC}+\mathrm{b}_{7} \mathrm{AG}(\mathrm{A}-\mathrm{G})+\mathrm{b}_{8} \mathrm{AC}(\mathrm{A}-\mathrm{C})+$ $\mathrm{b}_{9} \mathrm{GC}(\mathrm{G}-\mathrm{C})+\mathrm{b}_{10} \mathrm{AGC}$

Y é a estimativa da resposta AA ou MRF e $b_{x}$ os coeficientes da equação e $\mathrm{A}, \mathrm{G}$ e $\mathrm{C}$ as proporções das matérias-primas presentes nas formulações, argila, LETA e CCA (composição A) e argila, CL e CCA (composição B).

\section{RESULTADOS E DISCUSSÃO}

Análise química dos materiais: na Tabela II são apresentados os resultados obtidos da caracterização química da argila, do LETA, da CL e da CCA.

Analisando quantitativamente os óxidos presentes nas amostras estudadas, verifica-se que a argila apresenta teores de $\mathrm{SiO}_{2}(49,83 \%)$ e $\mathrm{Al}_{2} \mathrm{O}_{3}(27,35 \%)$ similares às argilas para cerâmica vermelha, conforme sugerido em [10]. O teor de ferro inferior a 7\% indica que a argila tem baixa coloração vermelha e teores de fundentes. A CCA apresenta elevado teor de $\mathrm{SiO}_{2}(87,54 \%)$, indicando ser de alta refratariedade e apresentar características de redutor de plasticidade da massa. O LETA apresenta teor de $\mathrm{Fe}_{2} \mathrm{O}_{3}$ superior a $10 \%$, indicando coloração vermelha na massa e elevado teor de fundente. A CL apresenta teores de $\mathrm{CaO}(43,32 \%), \mathrm{MgO}$ $(7,36 \%)$ e $\mathrm{K}_{2} \mathrm{O}(8,24 \%)$, indicando ser fundente e formadora de poros para temperatura superior a $1000{ }^{\circ} \mathrm{C}$.

Granulometria da argila e dos resíduos: na Tabela III é apresentada a distribuição de tamanho de partículas das matérias-primas estudadas.

Verifica-se que as matérias-primas estudadas apresentaram comportamento, do ponto vista granulométrico, completamente distinto. A distribuição granulométrica da CCA é mais grosseira do que a argila e os outros tipos de resíduos, por apresentar apenas $0,82 \%$ abaixo de $2 \mu \mathrm{m}$. Em relação ao diâmetro médio de partícula, verifica-se que o resíduo do LETA apresenta maior tamanho médio de partículas $(62,33 \mu \mathrm{m})$, enquanto a argila apresenta um menor diâmetro $(11,31 \mu \mathrm{m})$. Este fato pode estar relacionado ao maior teor do argilomineral caulinita presente na argila. Verifica-se, da Tabela III, que a argila é composta por $24,06 \%$ (fração argila), $74,49 \%$ (silte) e $1,45 \%$ (areia) Comparando os 
Tabela II - Composição química dos resíduos e da argila comercial utilizada (\% em peso). [Table II - Chemical compositions of the wastes and commercial clay used (wt.\%).]

\begin{tabular}{cccccccccccccc}
\hline Amostra & $\mathrm{PF}$ & $\mathrm{SiO}_{2}$ & $\mathrm{Al}_{2} \mathrm{O}_{3}$ & $\mathrm{Fe}_{2} \mathrm{O}_{3}$ & $\mathrm{P}_{2} \mathrm{O}_{5}$ & $\mathrm{MgO}$ & $\mathrm{TiO}_{2}$ & $\mathrm{~K}_{2} \mathrm{O}$ & $\mathrm{CaO}$ & $\mathrm{MnO}$ & $\mathrm{CuO}$ & $\mathrm{V}_{2} \mathrm{O}_{5}$ & Total \\
\hline Argila & 16,48 & 49,83 & 27,35 & 2,72 & 0,00 & 0,00 & 0,86 & 2,56 & 0,00 & 0,03 & 0,00 & 0,04 & 99,87 \\
CCA & 5,82 & 87,54 & 0,00 & 0,11 & 1,75 & 0,00 & 0,00 & 3,18 & 1,25 & 0,29 & 0,02 & 0,00 & 99,96 \\
LETA & 30,12 & 21,12 & 35,96 & 10,32 & 0,19 & 0,00 & 0,93 & 0,89 & 0,26 & 0,03 & 0,01 & 0,05 & 99,88 \\
CL & 32,37 & 2,29 & 1,74 & 0,46 & 3,77 & 7,36 & 0,00 & 8,24 & 43,32 & 0,08 & 0,00 & 0,00 & 99,62 \\
\hline
\end{tabular}

Tabela III - Distribuição de tamanho de partículas das matérias-primas estudadas (Argila, LETA, CL e CCA). [Table III-Particle size distribution of red Clay and wastes.]

\begin{tabular}{|c|c|c|c|c|}
\hline \multirow{2}{*}{ Amostra } & \multicolumn{3}{|c|}{ Distribuição Granulométrica (\%) } & \multirow{2}{*}{$\begin{array}{c}\text { Diâmetro } \\
\text { Médio } \\
(\mu \mathrm{m})\end{array}$} \\
\hline & $(<2 \mu \mathrm{m})$ & $(2 \leq \mathrm{X}<65 \mu \mathrm{m})$ & $(65 \leq X<2000 \mu \mathrm{m})$ & \\
\hline Argila & 24,06 & 74,49 & 1,45 & 11,31 \\
\hline $\mathrm{CCA}$ & 0,82 & 75,21 & 23,97 & 47,59 \\
\hline $\begin{array}{l}\text { Lodo de } \\
\text { ETA }\end{array}$ & 5,50 & 52,79 & 41,71 & 62,33 \\
\hline CL & 8,44 & 87,49 & 4,04 & 25,62 \\
\hline
\end{tabular}

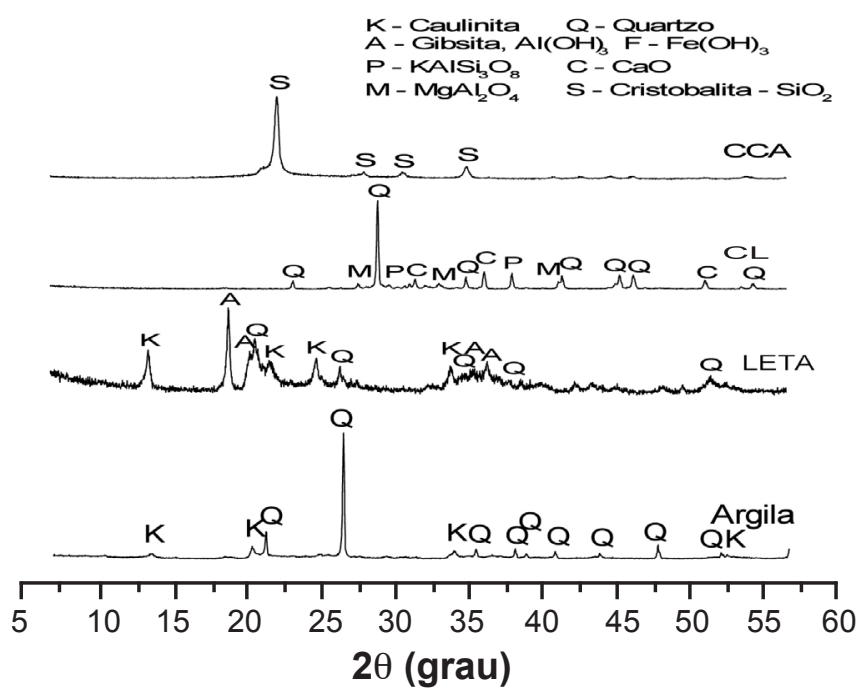

Figura 3: Difratogramas de raios $\mathrm{X}$ da argila e dos resíduos.

[Figure 3: X-ray diffraction patterns of the red clay and waste.]

valores de distribuição granulométrica da argila com os resultados obtidos por [10] verifica-se que são similares.

Análise por difração de raios $X$ da argila e dos resíduos: os difratogramas de raios X para a CCA, a CL, o LETA e a argila são apresentados na Fig. 3 .

Verifica-se que a argila é constituída de caulinita e quartzo $\left(\mathrm{SiO}_{2}\right)$, o LETA por caulinita, quartzo $\left(\mathrm{SiO}_{2}\right)$ e gibsita $\mathrm{Al}(\mathrm{OH})_{4}$, a CL por quartzo, aluminato de magnésio $\left(\mathrm{MgAl}_{2} \mathrm{O}_{4}\right)$, feldspato potássico e óxido de cálcio $(\mathrm{CaO})$ e a CCA é por sílica $\left(\mathrm{SiO}_{2}\right)$.

Sobreposição entre a absorção e o módulo de ruptura à flexão: a Fig. 4 apresenta as sobreposições das superfícies de resposta da absorção de água e do módulo de ruptura

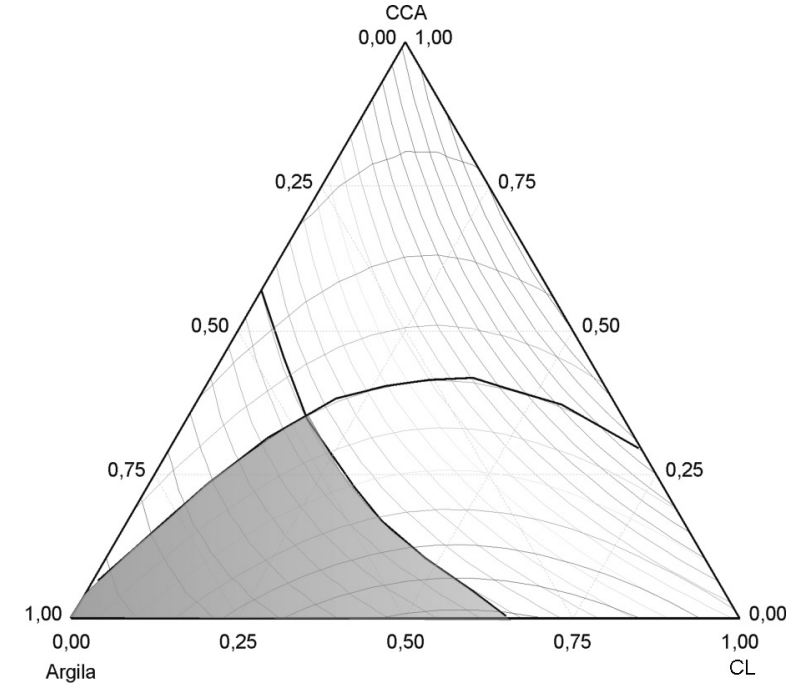

Figura 4: Sobreposição das superfícies de resposta da absorção de água e do módulo de ruptura após queima a $800^{\circ} \mathrm{C}$.

[Figure 4: Intersection of the water absorption and modulus of rupture response surface after firing at $800^{\circ} \mathrm{C}$.]

à flexão após a queima a $800{ }^{\circ} \mathrm{C}$ para a composição B. Já para a composição A na temperatura de $800{ }^{\circ} \mathrm{C}$, nenhuma mistura no triângulo estudado apresentou MRF superior a $5 \mathrm{MPa}$. Pode-se observar a região (região hachurada) onde as absorções e os módulos de ruptura são adequados aos valores previstos na normalização [11] para a produção de blocos cerâmicos, conforme a temperatura de queima.

Após queima a $800{ }^{\circ} \mathrm{C}$ é possível produzir blocos cerâmicos utilizando teores consideráveis de resíduos, como, por exemplo, peças contendo $25 \%$ de CL e $8,33 \%$ de CCA.

Com base na Fig. 5, verifica-se que a região do diagrama argila-resíduo para a composição A ficou reduzida com a queima a $900{ }^{\circ} \mathrm{C}$, e que para a composição B manteve-se quase constante comparativamente à queima a $800{ }^{\circ} \mathrm{C}, \mathrm{o}$ que indica a possibilidade de se incorporar resíduo de CL e CCA produzindo corpos com absorções inferiores e maiores módulos de ruptura.

Verifica-se que a região hachurada para a composição A teve um incremento com a queima a $1000{ }^{\circ} \mathrm{C}$, e que para a composição B houve um aumento da mesma, comparativamente à queima a $900{ }^{\circ} \mathrm{C}$, o que indica ser possível incorporar elevados teores de resíduo produzindo corpos com absorções inferiores e maiores módulos de ruptura. 

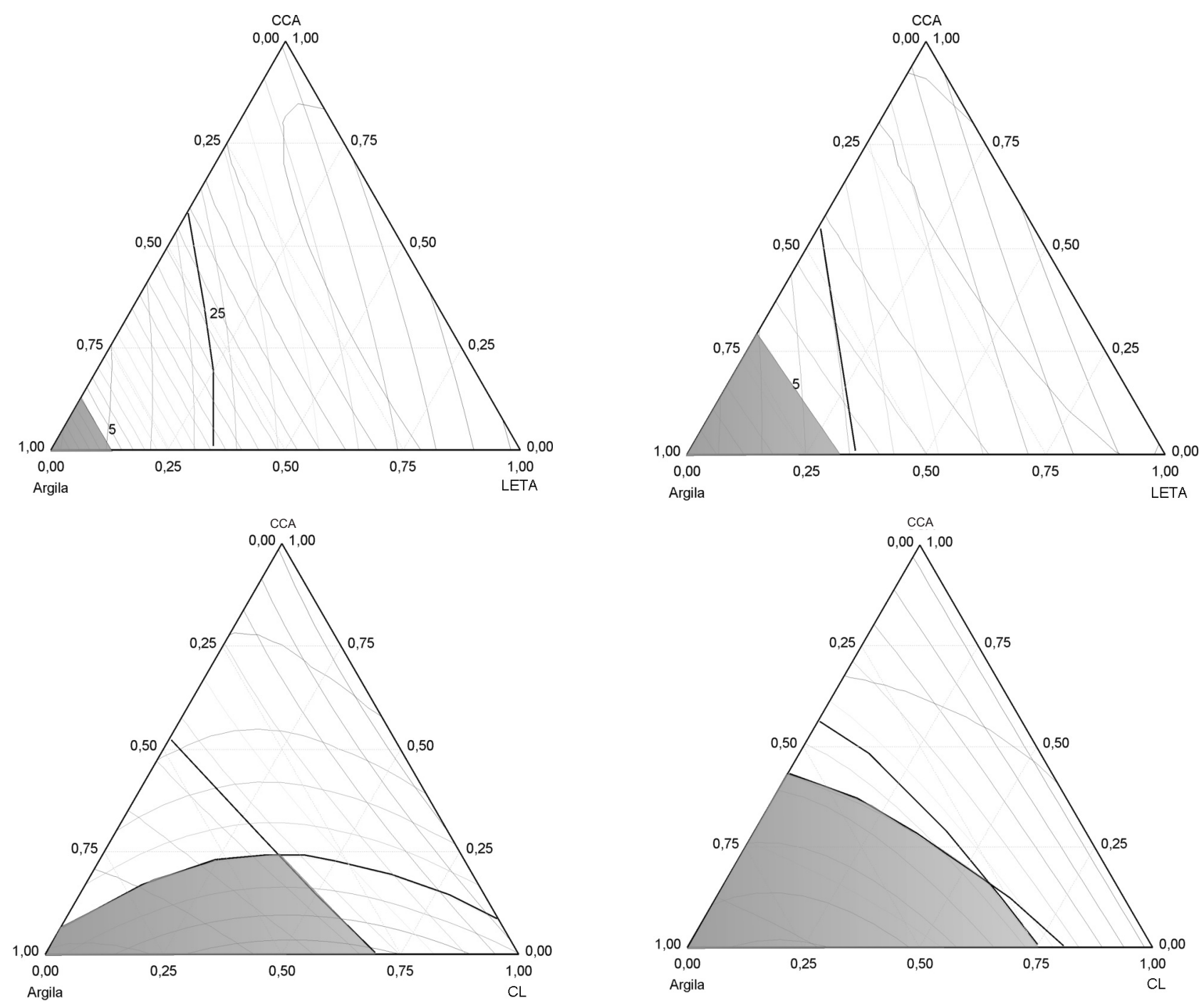

Figura 5: Sobreposição das superfícies de resposta da absorção de água e do módulo de ruptura após queima a $900{ }^{\circ} \mathrm{C}$.

[Figure 5: Intersection of the water absorption and modulus of rupture response surface after firing at $900^{\circ} \mathrm{C}$.]

\section{CONCLUSÕES}

A temperatura de $800{ }^{\circ} \mathrm{C}$ para a composição B mostrou que o incremento na quantidade de CL pode reduzir a AA. Na temperatura de $900{ }^{\circ} \mathrm{C}$ para a composição A, percebeuse uma discreta diminuição da AA com adição de CCA, e um aumento da mesma com a adição de LETA. No entanto, quando se realiza a queima a $1000^{\circ} \mathrm{C}$, observa-se que a adição dos resíduos, analisando em teores fixos de argila, aumenta a AA. Os valores mais elevados do MRF correspondem às composições com altas quantidades de argila (>90\%) para as temperaturas de $800{ }^{\circ} \mathrm{C}, 900{ }^{\circ} \mathrm{C}$ e $1000{ }^{\circ} \mathrm{C}$ na composição $\mathrm{A}$. Já para a composição $\mathrm{B}$ nas temperaturas de $800{ }^{\circ} \mathrm{C}$ e $900{ }^{\circ} \mathrm{C}$, observou-se que os valores elevados do MRF estão associados ao incremento das quantidades de argila e CL. O aumento da quantidade de CCA gera uma diminuição de MRF em todas as temperaturas. A CL

Figura 6: Sobreposição das superfícies de resposta da absorção de água e do módulo de ruptura após queima a $1000{ }^{\circ} \mathrm{C}$.

[Figure 6: Intersection of the water absorption and modulus of rupture response surface after firing at $1000^{\circ} \mathrm{C}$.]

melhora o desempenho dos corpos quando da queima a 800 e $900{ }^{\circ} \mathrm{C}$, visto que apresenta elevadas quantidades de fundentes tais como $\mathrm{P}_{2} \mathrm{O}_{5}, \mathrm{MgO}, \mathrm{K}_{2} \mathrm{O}$ e $\mathrm{CaO}$, o que pode favorecer o desenvolvimento de fases líquidas em baixas temperaturas (através de formação de eutéticos), sendo interessantes para as indústrias de cerâmica vermelha, pois estas queimam a baixas temperaturas. E também evidencia que, nesse caso, os maiores MRF não estão relacionados com menores AA, possivelmente devido a características microestruturais do sistema (como novas fases cristalinas, por exemplo). Conclui-se que através das técnicas empregadas, foi possível selecionar as melhores combinações das três argilas para produzir um tijolo estrutural com propriedades específicas.

\section{AGRADECIMENTOS}

Ao $\mathrm{CNPq}$ pelo apoio financeiro e ao Laboratório de 
Cerâmica do Departamento de Engenharia de Materiais da Universidade Federal de Campina Grande, PB pela realização de todos os ensaios.

\section{REFERÊNCIAS}

[1] M. A. Cincotto, "Seleção de materiais quanto à atividade pozolânica”, Tecnologia de Edificações, Ed. IPT, S. Paulo, SP 1 (1988) 23-26.

[2] Instituto Brasileiro de Geografia e Estatística - IBGE, disponível em: http://www.ibge.gov.br/home/estatistica/ indicadores/agropecuaria/lspa/lspa_20090_7.shtm, acesso 17/03/2009.

[3] L. R. Prudêncio Jr., S. Santos, D. A. Dafico, "Estudos com cinzas de casca de arroz buscam aproveitamento em concretos de alto desempenho", Coletânea Habitare: Utilização de resíduos na construção civil, Ed. Programa de Tecnologia de Habitação, 4 (2002).

[4] P. K. Mehta, "Rice Husk Ash: A Unique Supplementary Cementing Material", Adv. Concrete Technol., Ottawa Canmet, Canadá (1992) 407-431.

[5] Food and agriculture organization of the United Nations - FAO, Disponível em: http://www.fao.org/rice2004/ en/ aboutiyr.htm, acesso 20/01/2009.
[6] K. F. Portella, C. V. Andreoli, C. Hoppen, A. Sales, O. Baron, "Caracterização físico-química do lodo centrifugado da estação de tratamento de água Passaúna", Curitiba, PR, Anais $22^{\circ}$ Cong. Bras. Eng. Sanit. Amb., Joinvile, SC (2003). [7] J. S. Cordeiro, "Importância do tratamento e disposição adequada dos lodos de ETAs", in M. A. P. Reali (coord.), "Noções gerais de tratamento de disposição final de lodos de ETA", ABES/PROSAB, Rio de Janeiro, RJ (1999) 1-19. [8] M. C. Borlini, C. M. F. Vieira, D. G. Pinatti, et al., "Análise microestrutural de cerâmica vermelha incorporada com cinza de lenha de eucalipto", Anais Cong. Bras. Ceram., Blumenau, SC (2006).

[9] P. Souza Santos, Tecnologia de argilas, aplicação às argilas brasileiras, S. Paulo, SP, Ed. Edgard Blucher, 2 (1992) $340 \mathrm{p}$.

[10] C. M. F. Vieira, J. N. F. Holanda, D. G. Pinatti, "Caracterização de massa cerâmica vermelha utilizada na fabricação de tijolos na região de Campos dos Goytacazes", RJ, Cerâmica 46, 297 (2000) 14.

[11] ABNT, Associação Brasileira de Normas Técnicas; Norma Técnica, NBR 13818, Placas cerâmicas para revestimento - especificação e métodos de ensaio (1967) 14-16.

(Rec. 28/01/2010, Rev. 12/04/2010, Ac. 13/04/2010) 